\title{
Compressed Sensing-Based Range-Doppler Processing Method for Passive Radar
}

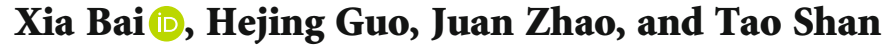 \\ School of Information and Electronics, Beijing Institute of Technology, Beijing 100081, China \\ Correspondence should be addressed to Xia Bai; bai@bit.edu.cn
}

Received 9 February 2021; Accepted 9 July 2021; Published 23 July 2021

Academic Editor: Liangtian Wan

Copyright ( 2021 Xia Bai et al. This is an open access article distributed under the Creative Commons Attribution License, which permits unrestricted use, distribution, and reproduction in any medium, provided the original work is properly cited.

\begin{abstract}
Passive radar (PR) systems use the existing transmitters of opportunity in the environment to perform tasks such as detection, tracking, and imaging. The classical cross-correlation based methods to obtain the range-Doppler map have the problems of high sidelobe and limited resolution due to the influence of signal bandwidth. In this paper, we propose a novel range-Doppler processing method based on compressed sensing (CS), which performs sparse reconstruction in range and Doppler dimensions to achieve high resolution and reduces sidelobe without excessive computational burden. Results from numerical simulations and experimental measurements recorded with the Chinese standard digital television terrestrial broadcasting (DTTB) based PR show that the proposed method successfully handles the range-Doppler map formatting problem for PR and outperforms the existing CS-based PR processing methods.
\end{abstract}

\section{Introduction}

Passive radar [1] (PR) is a kind of radar system which uses the existing transmitters of opportunity (such as FM $[2,3]$, GSM [4], and DVB-T [5, 6]) in space to achieve target detection and tracking and other tasks without special deployment or installation of transmitters. In recent years, PR has been widely concerned in the military and commercial fields because of advantages in terms of low-cost implementation, confidentiality, strong antijamming, and reduced electromagnetic pollution to the environment.

In this study, we mainly focus on the generation of a passive radar range-Doppler (RD) map. Based on the matched filtering theory, the classical method uses the crossambiguity function (CAF) and fast Fourier transform (FFT) to calculate $\mathrm{RD}$ response. However, the classical method faces some challenges. On the one hand, the generated $\mathrm{RD}$ map has a high sidelobe level, which may get false target position and Doppler frequency. On the other hand, the signal bandwidth of passive radar is usually narrow compared to that of active radar. It limits range resolution, and this limitation leads to undesirable performance in various applications [7]. In order to improve the range resolution of PR, the mul- tiple broadcast channels from a single transmitter were exploited [8-10], which can be implemented only when the multiband system is used. Besides, a longer integration time can improve the Doppler resolution. But that would cause migration phenomena during integration and require complex compensation $[11,12]$.

In recent years, the application of compressed sensing (CS) has been considered in passive radar. CS is a sparse signal processing technology $[13,14]$, which can reduce the amount of sampling data and use a small number of measurements to achieve excellent signal reconstruction. CS has been widely used in the field of radar signal processing; it has great potential in improving resolution $[15,16]$. With the increasing attention in the field of $\mathrm{PR}$, the $\mathrm{RD}$ map of targets for PR can be formulated as a sparse recovery problem, and the works related to CS-based PR processing have been published [17-19]. In [17, 18], a normal CS-based PR processing scheme was proposed to achieve better range and Doppler resolutions. Due to the fact that the dictionary is composed of the template signals with discrete delays and Doppler shifts, this method has high reconstruction accuracy, but it needs a large amount of storage and calculations. [19] proposed a RD map generation algorithm for PR. The 
extended orthogonal matching pursuit (EOMP) algorithm is employed to obtain the Doppler frequency with an improved resolution and a reduced sidelobe level. But one-dimensional cross-correlation is used to obtain the range compressed profile, which is the same as the classical method.

In this paper, we propose a novel range-Doppler processing method for PR. CS is applied to the range domain and Doppler domain, respectively, which no longer requires huge storage space occupied by the dictionary. In addition, our contributions are the following. (1) In the Doppler dimension, considering the sparse characteristics of the signal, we present a modified OMP algorithm based on the multiple measurement vector (MMV) [20] model to improve the processing ability. (2) In the range dimension, we use the Fourier dictionary in the range-frequency domain, which can easily achieve high-resolution range estimation. Furthermore, a global search is to find the most relevant atom of the dictionary matrix for sparse reconstruction of the $\mathrm{RD}$ map, which can be treated as another way to use EOMP.

The rest of this paper is organised as follows. A brief signal model of PR is introduced in Section 2. In Section 3, a novel theoretical derivation of CS-based RD map generation for $\mathrm{PR}$ is presented. The comparison with other processing methods is also discussed in this section. Section 4 demonstrates experimental results using the simulated data and the real data. Finally, Section 5 concludes this work.

\section{Passive Radar Geometry and Signal Model}

Figure 1 schematically illustrates a typical passive radar geometry, where the system is composed of transmitting station $T$ and receiving station $R$. It is assumed that there is a moving target $P$ in the scene. $R_{T}, R_{R}$, and $R_{L}$ represent the transmitter-target, receiver-target, and transmitter-receiver distances. $v$ is the velocity vector of the moving target. $\beta$ is the bistatic angle. $\phi$ is the angle between the vector $v$ and the bistatic angle. $\theta_{T}$ is the emission angle, and $\theta_{R}$ is the target observation angle.

The $\mathrm{PR}$ receiver consists of two channels, the reference channel and surveillance channel. The reference channel gathers a time-delayed version of the transmitted waveform, and the surveillance channel records the signals scattered from targets. Let the transmitted waveform be represented by $x_{T}(t)$, then the signal collected by the reference channel can be written as

$$
x(t)=A_{r} x_{T}\left(t-\tau_{L}\right)+n_{r}(t)
$$

where $A_{r}$ is the complex amplitude, $\tau_{L}=R_{L} / c$ represents the time delay, and $n_{r}(t)$ represents the thermal noise in the reference channel.

Admittedly, the surveillance channel also contains direct signal and multipath in practice. Here, we assume that disturbance has been removed [21-24], and then, the response of the moving target $P$ can be expressed as

$$
y(t)=A x_{T}\left(t-\tau_{p}\right) \exp \left(j 2 \pi f_{p} t\right)+n(t)
$$

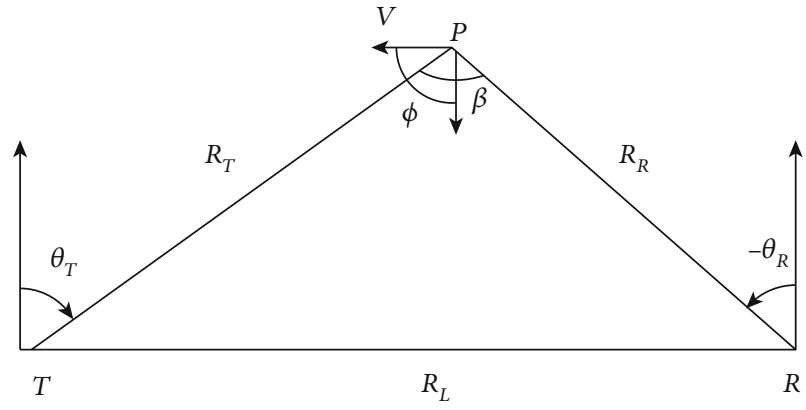

FIgURE 1: Simplified bistatic geometry for passive radar.

where $A$ is the complex amplitude, $\tau_{p}$ is the bistatic time delay corresponding to the target location, $f_{p}$ is the Doppler frequency shift related to the target velocity, and $n(t)$ is the thermal noise in the surveillance channel. According to the geometric relationship between the target and the bistatic radar system, the time delay and instantaneous Doppler frequency of the target can be expressed as [25]

$$
\begin{aligned}
& \tau_{p}=\frac{\sqrt{R_{R}^{2}+R_{L}^{2}+2 R_{R} R_{L} \sin \theta_{R}}+R_{R}}{c}, \\
& f_{p}=\frac{2 v}{\lambda} \cos (\phi) \cos \left(\frac{\beta}{2}\right) .
\end{aligned}
$$

Considering an observation scene consisting of $K$ scattering points, a generalized expression of the surveillance signal can be written as

$$
y(t)=\sum_{k=1}^{K} A_{k} x_{T}\left(t-\tau_{k}\right) \exp \left(j 2 \pi f_{k} t\right)+n(t),
$$

where $A_{k}, f_{k}$, and $\tau_{k}$ are the complex amplitude, the bistatic time delay, and the Doppler frequency shift of the $k$ th scattering point. To simplify the analysis, the thermal fluctuations $n_{r}(t)$ and $n(t)$ are neglected, $A_{r}$ is set to 1 , and $\tau_{L}$ is set to 0 . Then, the surveillance signal can be rewritten as

$$
y(t)=\sum_{k=1}^{K} A_{k} x\left(t-\tau_{k}\right) \exp \left(j 2 \pi f_{k} t\right)
$$

It should be noted that this simplification has no significant impact. For example, the condition $\tau_{L}=0$ can be satisfied as long as the reference signal is added a corresponding time delay.

\section{CS-Based Processing for PR}

In this section, we present our investigation of the following CS-based method to form a range-Doppler map with passive radar data.

3.1. Signal Preprocessing. The surveillance signal and the reference signal first need to be divided into multiple short segments [26], as shown in Figure 2. It means that the Doppler frequency change within a segment interval is ignored. 


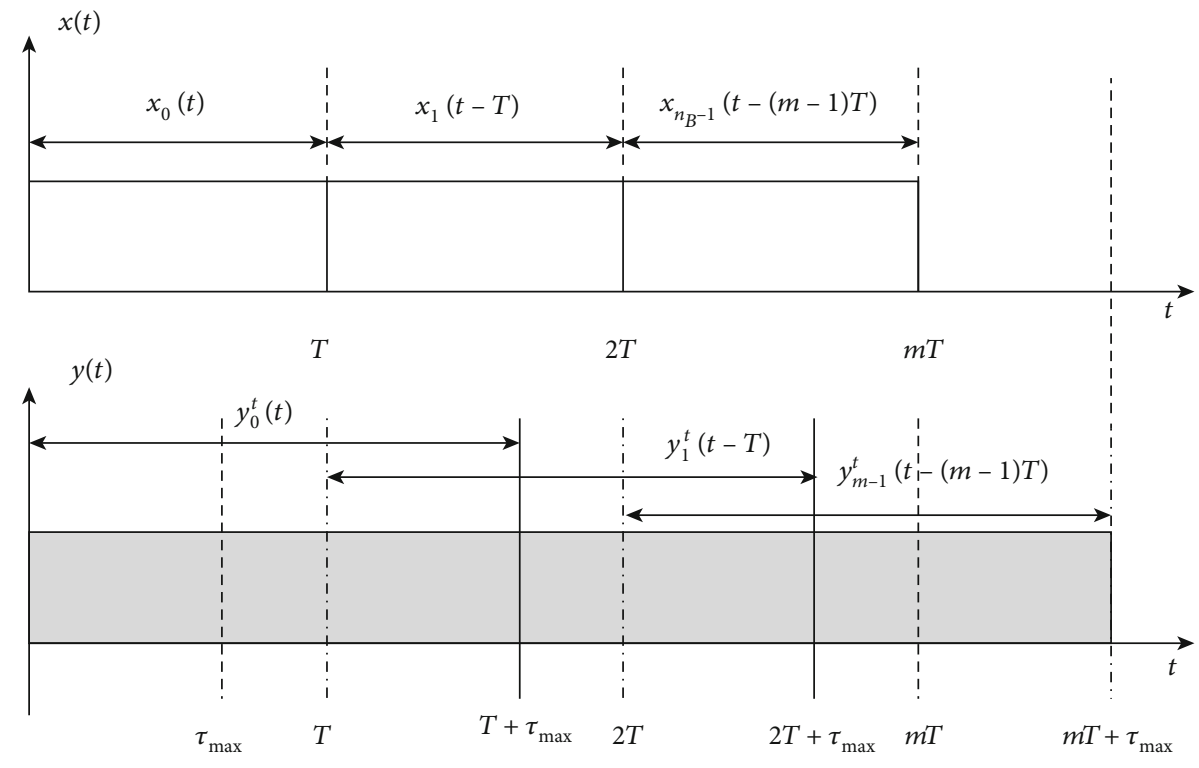

Figure 2: Signal segmentation.
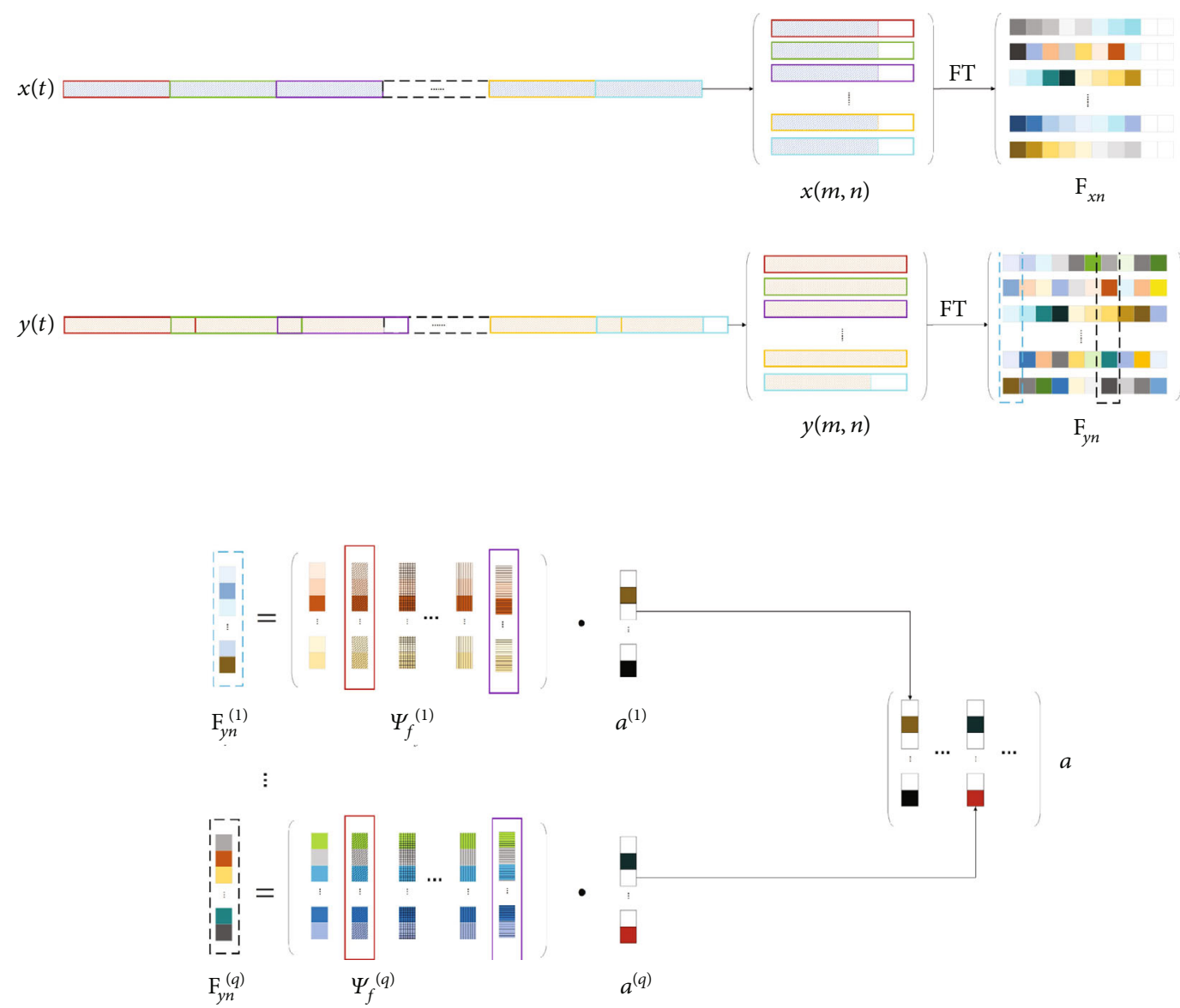

FIGURE 3: MMV model for passive radar. 


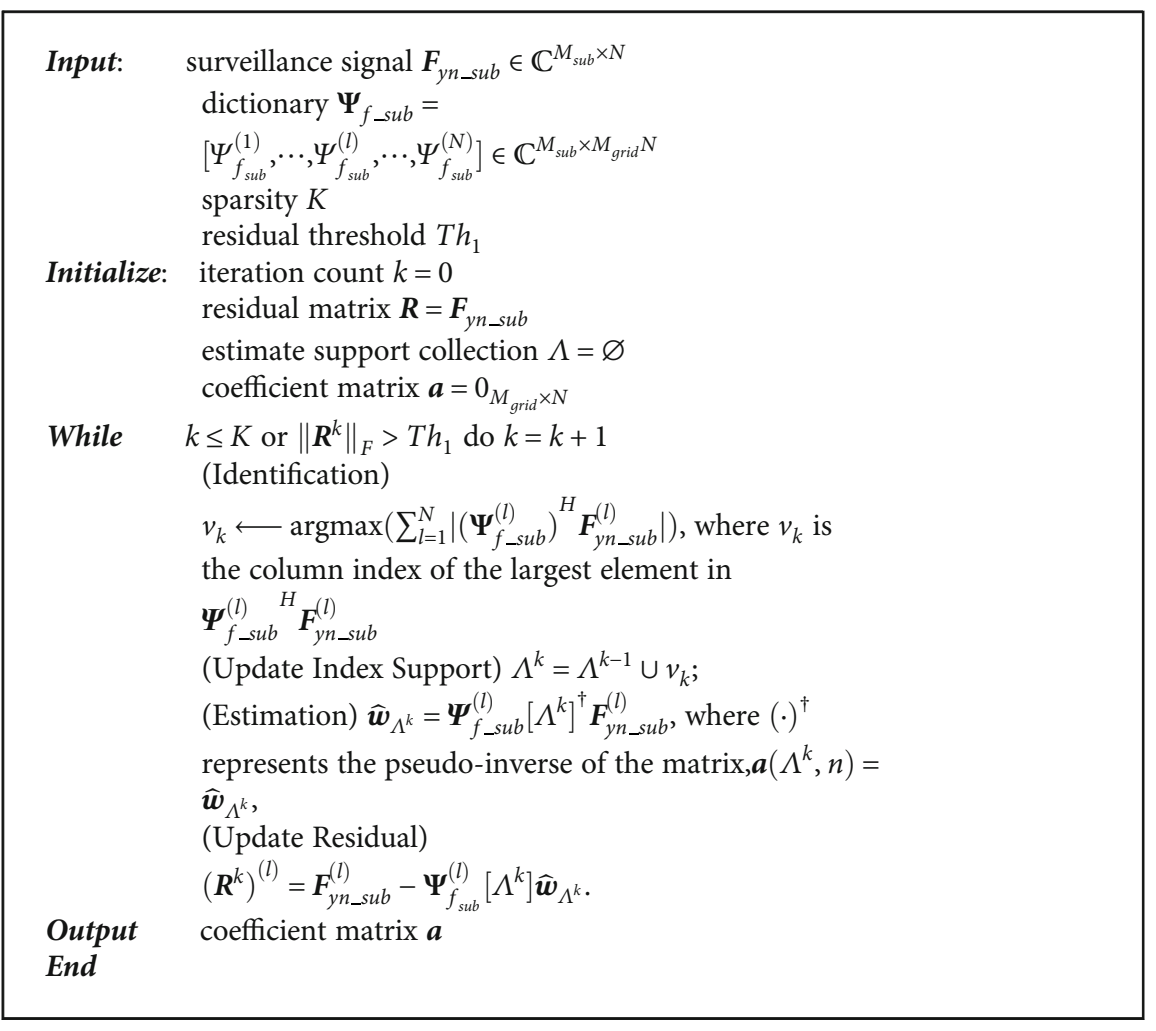

Algorithm 1: Pseudocode of modified MMV-OMP algorithm for Doppler reconstruction.

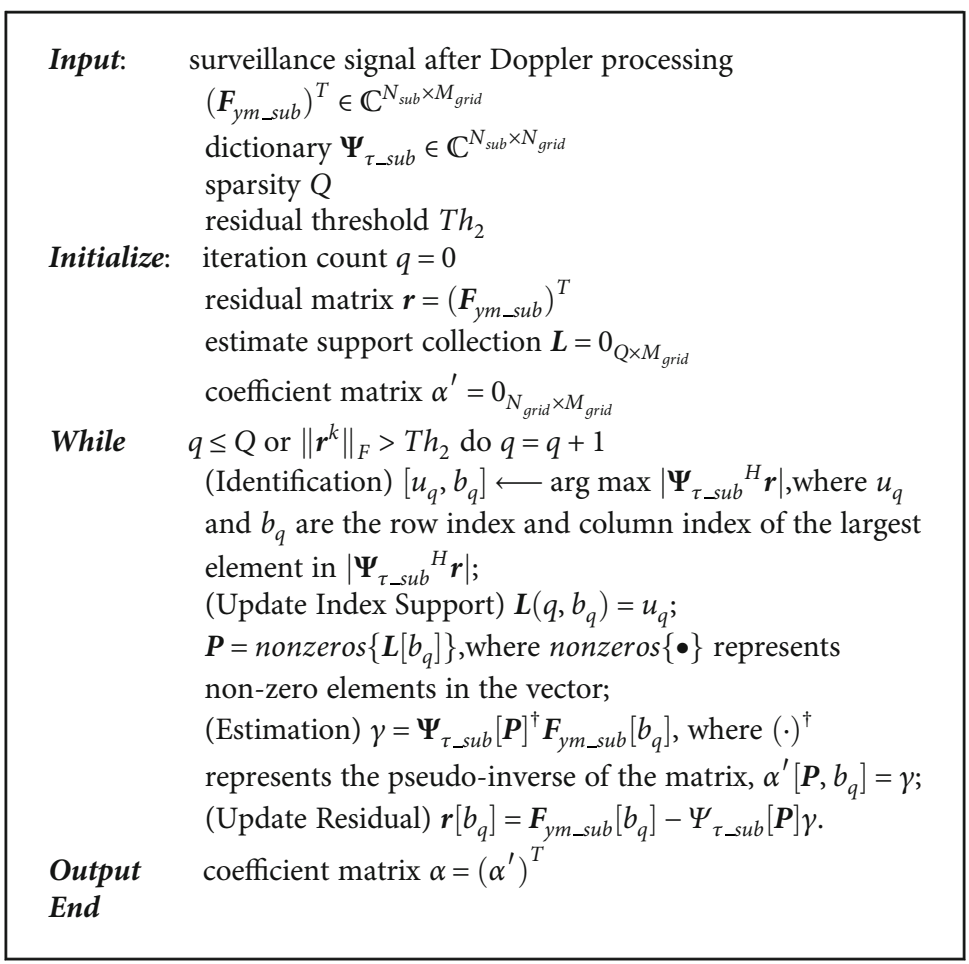

Algorithm 2: Pseudocode of EOMP algorithm for range reconstruction. 


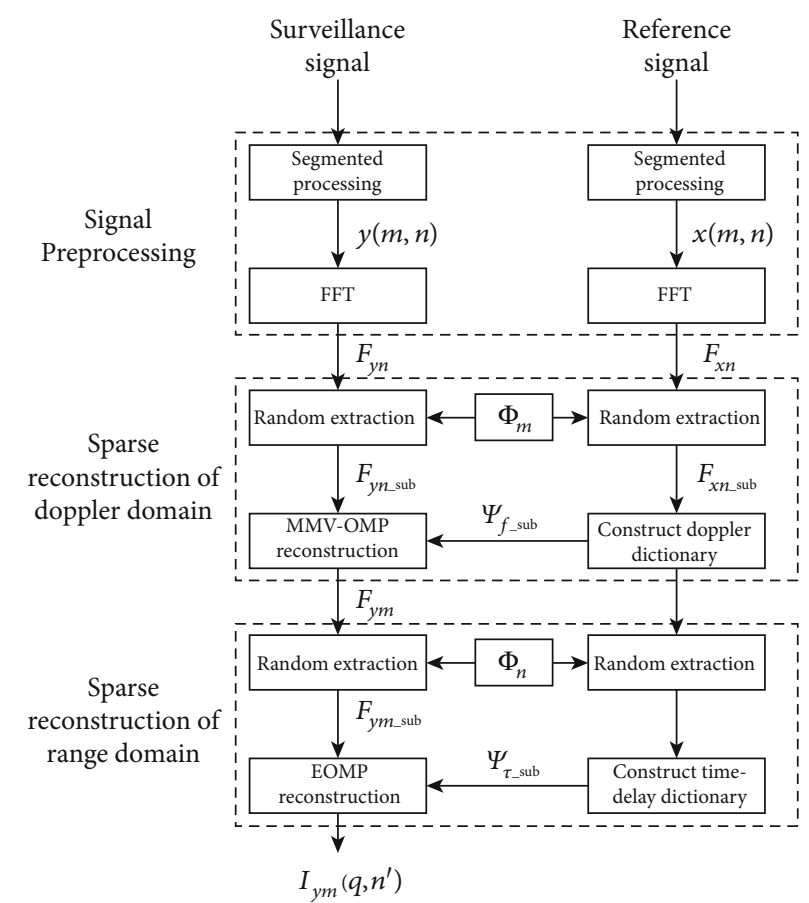

Figure 4: Flowchart of the proposed method.

TABLE 1: CS-PR method comparison.

\begin{tabular}{|c|c|c|c|}
\hline Methods & Original method & Feng et al.'s method & Proposed method \\
\hline Signal model & $r D: \mathbf{y}_{\mathrm{vec}}=\boldsymbol{\Psi}_{r D} \boldsymbol{\alpha}_{\mathrm{vec}}$ & $D: \mathbf{y}_{r}=\boldsymbol{\Psi}_{D} \boldsymbol{\alpha}$ & $\begin{aligned} D & : \mathbf{F}_{y n_{\text {_sub }}}{ }^{(l)}=\boldsymbol{\Psi}_{f \_ \text {sub }}^{(l)} \mathbf{a}^{(l)} \\
r: \mathbf{F}_{y m_{\text {sub }}} & =\boldsymbol{\Psi}_{\tau_{\text {_sub }}} \boldsymbol{\alpha}\end{aligned}$ \\
\hline Dictionary size & $\begin{array}{c}\Psi_{\mathrm{rD}} \\
\left(M_{\text {sub }} N_{\text {sub }} \times M_{\text {grid }} N_{\text {grid }}\right)\end{array}$ & $\Psi_{D}\left(M_{\text {sub }} \times M_{\text {grid }}\right)$ & $\begin{array}{c}D: \Psi_{f_{\text {sub }}}^{(l)}\left(M_{\text {sub }} \times M_{\text {grid }}\right) \\
r: \Psi_{\tau_{\text {sub }}}\left(N_{\text {sub }} \times N_{\text {grid }}\right)\end{array}$ \\
\hline
\end{tabular}

Let $t=\tau+m T$ (where $T$ is the segment interval); $t$ can be called slow time. $\tau$ represents time delay, which is called fast time. Now, the surveillance signal is expressed as follows:

$$
\begin{aligned}
y(\tau) & =\sum_{k=1}^{K} A_{k} x\left(\tau-\tau_{k}+m T\right) \exp \left(j 2 \pi f_{k} \tau\right) \exp \left(j 2 \pi f_{k} m T\right) \\
& \approx \sum_{k=1}^{K} A_{k} x\left(\tau-\tau_{k}+m T\right) \exp \left(j 2 \pi f_{k} m T\right)
\end{aligned}
$$

where $m=0,1, \cdots, M-1$ is the slow time index and $M$ denotes the number of segments. The two-dimensional discrete form of (6) can be expressed as

$$
y(m, n)=\sum_{k=1}^{K} A_{k} x\left(n \Delta_{\tau}-n_{k} \Delta_{\tau}+m T\right) \exp \left(j 2 \pi f_{k} m T\right),
$$

where $\Delta_{\tau}$ is the sampling time interval and $\tau_{k}=n_{k} \Delta_{\tau} . n=0$ $, 1, \cdots, N-1$ is the fast time index, and $N$ denotes the sample number of each segment. Similarly, the discrete representation of the reference signal is expressed as

$$
x(m, n)=x\left(n \Delta_{\tau}+m T\right) .
$$

3.2. Sparse Reconstruction of Doppler Domain. By taking an FFT of $x(m, n)$ with respect to $n$, we have

$$
F_{x n}(m, l)=\sum_{n=0}^{N-1} x(m, n) \exp \left(-j 2 \pi l \Delta f_{n} n \Delta_{\tau}\right)=\sum_{n=0}^{N-1} x(m, n) \exp \left(-j l \frac{2 \pi}{N} n\right)
$$

where $\Delta f_{n}=1 /\left(N \Delta_{\tau}\right)$ is the range-frequency bin size and $l$ is the range-frequency index. Similarly, after performing an FFT of $y(m, n)$ with respect to $n$, we can obtain

$$
\begin{aligned}
F_{y n}(m, l) & =\sum_{n=0}^{N-1} y(m, n) \exp \left(-j 2 \pi l \Delta f_{n} n \Delta_{\tau}\right) \\
& =\sum_{k=1}^{K} A_{k} F_{x n}(m, l) \exp \left(-j 2 \pi l \Delta f_{n} n_{k} \Delta_{\tau}\right) \exp \left(j 2 \pi f_{k} m T\right) \\
& =\sum_{k=1}^{K} A_{k} F_{x n}(m, l) \exp \left(-j l \frac{2 \pi}{N} n_{k}\right) \exp \left(j 2 \pi f_{k} m T\right) .
\end{aligned}
$$




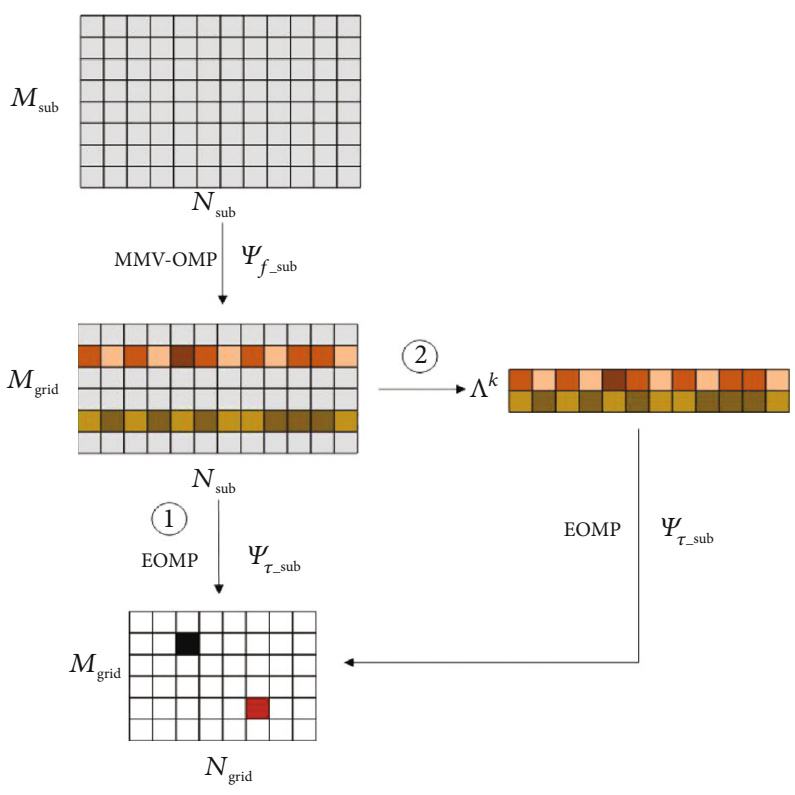

FIGURE 5: The diagram of signal reconstruction.

In order to make a sparse representation of the rangefrequency bins, a Doppler dictionary $\Psi_{f}^{(l)} \in \mathbb{C}^{M \times M_{\text {grid }}}$ is constructed as

$$
\begin{aligned}
& \boldsymbol{\Psi}_{f}^{(l)}=\left[\begin{array}{lllll}
\psi_{1}^{(l)} & \cdots & \psi_{q}^{(l)} & \cdots & \psi_{M_{\text {grid }}}^{(l)}
\end{array}\right], \\
& \psi_{q}^{(l)}=F_{x n}(m, l) \exp \left(j \frac{2 \pi}{M_{\text {grid }}} q m\right), \quad m=0,1, \cdots, M-1,
\end{aligned}
$$

where $M_{\text {grid }}$ denotes the number of the Doppler grid. And then, (10) can be expressed as

$$
F_{y n}(m, l)=\sum_{k=1}^{K} A_{k} F_{x n}(m, l) \exp \left(-j l \frac{2 \pi}{N} n_{k}\right) \exp \left(j \frac{2 \pi}{M_{\text {grid }}} q_{k} m\right)
$$

where $q_{k}=f_{k} / \Delta f_{q}$ and $\Delta f_{q}=1 /\left(M_{\text {grid }} T\right)$ represent the size of the Doppler grid. From this, we may know that $M_{\text {grid }}=M$ makes the same resolution level as the classical method, $\Delta$ $f_{q}=\Delta f=1 /(M T)$, and the larger $M_{\text {grid }}$ can generate higher Doppler resolution.

For each range-frequency bin $l,(12)$ can be rewritten as

$$
\mathbf{F}_{y n}^{(l)}=\Psi_{f}^{(l)} \mathbf{a}^{(l)},
$$

where

$$
\begin{aligned}
\mathbf{F}_{y n}{ }^{(l)} & =\left[\begin{array}{lllll}
F_{y n}(0, l) & \cdots & \cdots & F_{y n}(M-1, l)
\end{array}\right]^{T}, \\
\mathbf{a}^{(l)} & =\left[\begin{array}{lllll}
a_{1} & \cdots & a_{q} & \cdots & a_{M_{\text {grid }}}
\end{array}\right]^{T} .
\end{aligned}
$$

TABLE 2: Frame structure of DTTB signal.

\begin{tabular}{lccc}
\hline & Frame head & System information & Data \\
\hline DTTB & 945 symbols & 36 symbols & 3744 symbols \\
\hline
\end{tabular}

TABLE 3: System parameter.

\begin{tabular}{lcc}
\hline Parameters & Symbol & Value \\
\hline Carrier frequency & $f_{c}$ & $674 \mathrm{MHz}$ \\
Bandwidth & $B$ & $7.56 \mathrm{MHz}$ \\
Sample frequency & $f_{s}$ & $10 \mathrm{MHz}$ \\
Number of segments & $M$ & 512 \\
Sample number & $N$ & 256 \\
Number of range grid & $M_{\text {grid }}$ & 50 \\
Number of Doppler grid & $N_{\text {grid }}$ & 20 \\
\hline
\end{tabular}

There are $K$ nonzero elements in $\mathbf{a}^{(l)}$, when the scattering points make different Doppler frequency shifts. And then, the positions and amplitude values are, respectively,

$$
\begin{aligned}
q_{k} & =\frac{f_{k}}{\Delta f_{q}}, \quad k=1,2, \cdots, K, \\
a_{q_{k}} & =A_{k} \exp \left(-j l \frac{2 \pi}{N} n_{k}\right), \quad k=1,2, \cdots, K .
\end{aligned}
$$

If there are the same Doppler frequencies, then the number of nonzero elements becomes smaller. In turn, the complex amplitude is the sum of all coefficients related to the same Doppler.

According to the CS theory, the sparsity of the signal ensures the feasibility of reducing the amount of data. The 
TABLE 4: Target scenario parameters.

\begin{tabular}{|c|c|c|c|c|}
\hline Parameters & Target 1 & Scatterer point 1 & $\begin{array}{c}\text { Target } 2 \\
\text { Scatterer point } 2\end{array}$ & Scatterer point 3 \\
\hline Target location index & 10 & 26 & 28 & 30 \\
\hline Doppler bin & 4 & -4 & -4 & -4 \\
\hline Amplitude & 1 & 0.7 & 0.9 & 1 \\
\hline
\end{tabular}

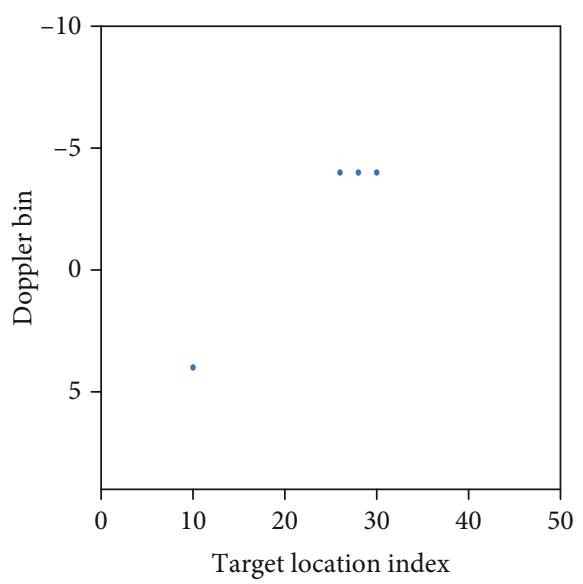

(a)

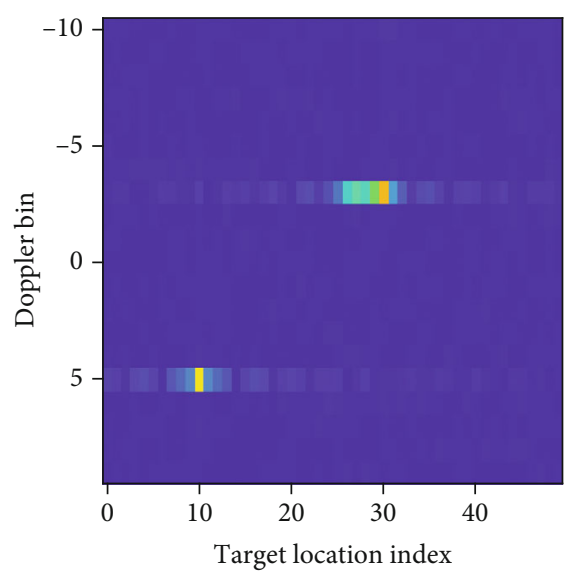

(b)

FIGURE 6: Result obtained by CAF: (a) the original scene; (b) RD map.

sensing matrix obtained by multiplying the measurement matrix $\Phi$ and the Doppler dictionary (sparse basis matrix) $\Psi_{f}^{(l)}$ needs to satisfy the restricted isometric property (RIP). The commonly used measurement matrices are the random Gaussian matrix and partial random unit matrix. In order to facilitate the practical operation, we adopt the method of random extraction of data, which can be expressed as

$$
\mathbf{F}_{y n_{-} \text {sub }}{ }^{(l)}=\boldsymbol{\Phi}_{m} \mathbf{F}_{y n}{ }^{(l)}=\boldsymbol{\Phi}_{m} \Psi_{f}^{(l)} \mathbf{a}^{(l)}=\Psi_{f \_s u b}^{(l)} \mathbf{a}^{(l)},
$$

where $\Phi_{m} \in \mathbb{R}^{M_{\text {sub }} \times M}$ is a partial random unit matrix. In order to obtain the coefficient vector $\mathbf{a}^{(l)}$, (16) needs to be solved. Due to the sparseness of the coefficient vector, it is equivalent to solving the following minimum norm problem:

$$
\mathbf{a} \wedge^{(l)}=\arg \min \left\|\mathbf{a}^{(l)}\right\|_{0} \text { s.t. } \mathbf{F}_{y n_{-} \text {sub }}{ }^{(l)}=\Psi_{f_{-} \mathrm{sub}^{(l)}}^{\mathbf{a}^{(l)}} .
$$

There are many methods to solve (17). The greedy algorithm is widely used in practical application because of its excellent geometric interpretation, good reconstruction effect, and fast reconstruction speed. The most representative greedy algorithm is the OMP algorithm. Considering the consistency of signal models of multiple range-frequency bins, the same operation can be carried out for each rangefrequency bin. That is to say, it can solve $\mathbf{a}^{(l)}$ through OMP under the single measurement vector (SMV) model according to $\mathbf{F}_{y n_{-} \text {sub }}{ }^{(l)}$ and $\Psi_{f \_ \text {sub }}^{(l)}$, respectively.

We note that 13 is independent of $l$. It is found that the positions of nonzero elements of $\mathbf{a}^{(l)}$ in multiple range- frequency bins are the same; that is, the support set of each sparse coefficient vector is the same. This feature means that it can be considered an MMV model (see Figure 3), and we can use the joint sparsity to improve reconstruction performance. However, the existing OMP algorithm under the MMV model cannot be directly applied. Considering that $\Psi_{f \_s u b}^{(l)}$ is different for passive radar data, a modified version of MMV-OMP is proposed here. The pseudocode is shown in Algorithm 1. In order to facilitate the derivation, the matrix $\mathbf{a}=\left[\mathbf{a} \wedge{ }^{(1)}, \cdots, \mathbf{a} \wedge^{(l)}, \cdots, \mathbf{a} \wedge^{(N)}\right]$ which is obtained by the modified MMV-OMP algorithm will be rewritten as $F_{y m}(q, l)$.

3.3. Sparse Reconstruction of Range Domain. After Doppler reconstruction, the two-dimensional data can be expressed as

$$
F_{y m}(q, l)= \begin{cases}\sum_{k} A_{k} \exp \left(-j l \frac{2 \pi}{N} n_{k}\right), & q=q_{k}, \\ 0, & q \neq q_{k} .\end{cases}
$$

It can be seen from (18) that the signal is independent of Doppler frequency bin $q$. Therefore, the same time-delay dictionary $\Psi_{\tau} \in \mathbb{C}^{N \times N_{\text {grid }}}$ can be constructed for each Doppler bin, which can be expressed as

$$
\begin{aligned}
& \boldsymbol{\Psi}_{\tau}=\left[\begin{array}{lllll}
\psi_{1} & \cdots & \psi_{n}{ }^{\prime} & \cdots & \psi_{N_{\text {grid }}}
\end{array}\right], \\
& \psi_{n}^{\prime}=\exp \left(-j \frac{2 \pi}{N_{\text {grid }}} n^{\prime} l\right), \quad l=0,1, \cdots, N-1 \text {, }
\end{aligned}
$$




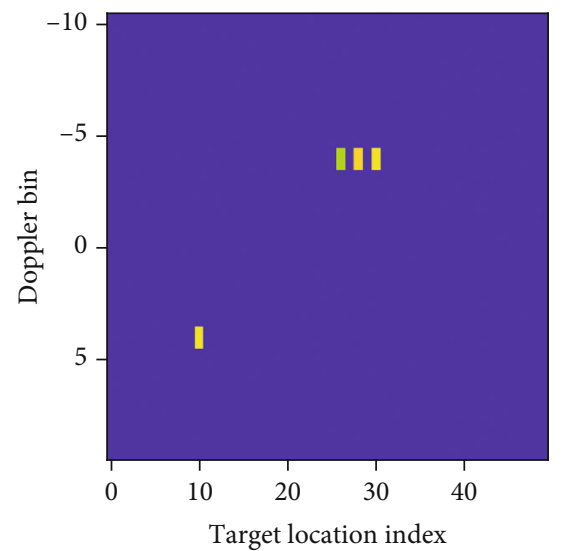

(a)

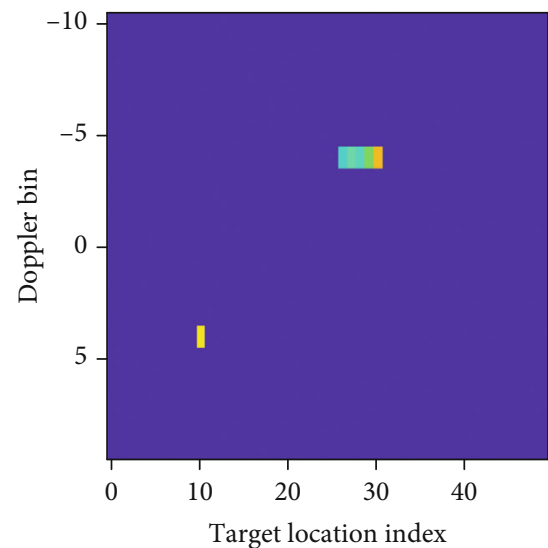

(c)

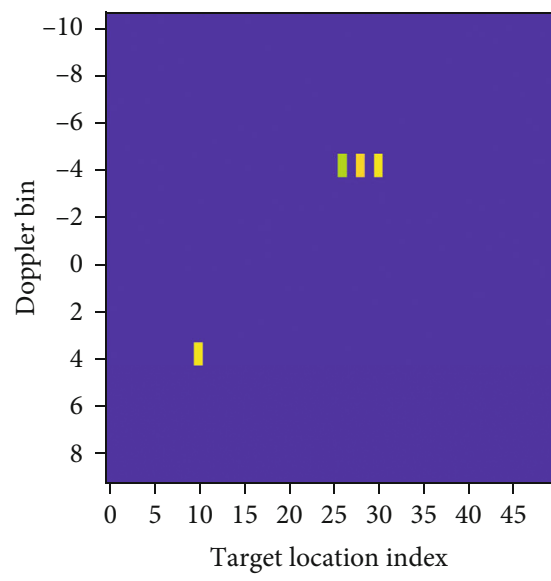

(e)

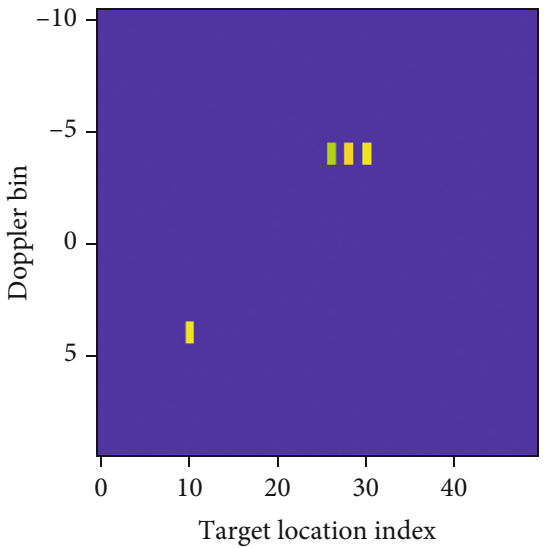

(b)

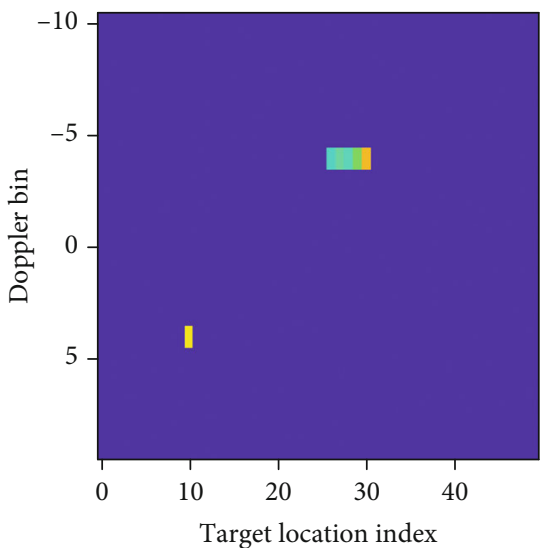

(d)

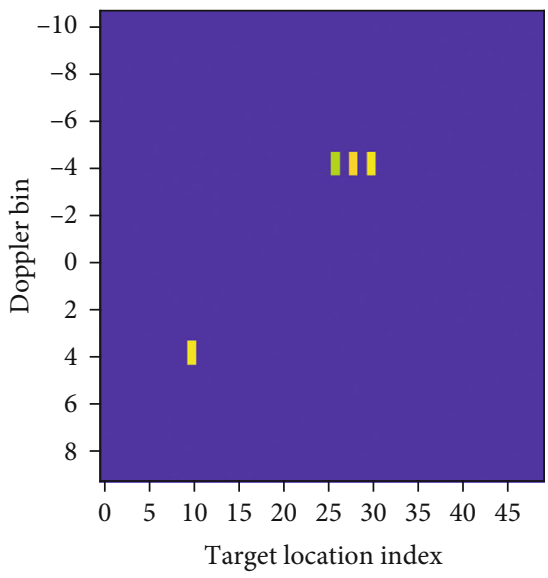

(f)

FIGURE 7: RD maps obtained by $(\mathrm{a}, \mathrm{b})$ original CS-PR method, $K=4$; (c, d) Feng et al.'s method, $K=4$; (e, f) proposed method, $K=Q=4$; (a, c, e) with full samples; (b, d, f) with partial samples (1/4).

where $N_{\text {grid }}$ denotes the number of time-delay (range) grids. So, the signal of $q$ th Doppler bin can be expressed as

$$
\mathbf{F}_{y m}{ }^{(q)}=\Psi_{\tau} \alpha^{(q)},
$$

where

$$
\begin{aligned}
\mathbf{F}_{y m}{ }^{(q)} & =\left[\begin{array}{lllll}
F_{y m}(q, 0) & \cdots & \cdots & F_{y m}(q, N-1)
\end{array}\right]^{T}, \\
\boldsymbol{\alpha}^{(q)} & =\left[\begin{array}{lllll}
\alpha_{1} & \cdots & \alpha_{n^{\prime}} & \cdots & \alpha_{N_{\text {grid }}}
\end{array}\right]^{T} .
\end{aligned}
$$

In whole range-Doppler plane, there are $K$ nonzero elements $\left(K<<M_{\text {grid }} N_{\text {grid }}\right)$, and the positions and amplitude values are, respectively, 


$$
\begin{gathered}
n^{\prime}{ }_{k}=\frac{\tau_{k}}{\Delta_{\tau}^{\prime}}, \quad k=1,2, \cdots, K, \\
\alpha_{n_{k}^{\prime}}=A_{k}, \quad k=1,2, \cdots, K,
\end{gathered}
$$

where $\Delta_{\tau}{ }^{\prime}=\Delta_{\tau}\left(N / N_{\text {grid }}\right)$. It was obvious that $N_{\text {grid }}=N$ makes the same or better resolution level as the classical method; the larger $N_{\text {grid }}$ can generate finer range resolution.

Similarly, the signal is randomly extracted, and the observation equation can be expressed as

$$
\mathbf{F}_{y m \_s u b}{ }^{(q)}=\boldsymbol{\Phi}_{n} \mathbf{F}_{y m}{ }^{(q)}=\boldsymbol{\Phi}_{n} \boldsymbol{\Psi}_{\tau} \boldsymbol{\alpha}^{(q)}=\boldsymbol{\Psi}_{\tau_{-} \text {sub }} \boldsymbol{\alpha}^{(q)},
$$

where $\Phi_{n} \in \mathbb{R}^{N_{\text {sub }} \times N}$ is a partial random unit matrix. It is important to note that the randomness has a constraint. For range-frequency bins, the frequency range is determined by the sampling rate $f_{s}$, which usually satisfies $f_{s} \geq B$ ( $B$ is the signal bandwidth). This means that some range-frequency bins contain invalid information. Therefore, the random extraction only considers the effective part of the signal bandwidth.

The coefficient vector $\boldsymbol{a}^{(q)}$ can be solved as follows:

$$
\alpha \wedge^{(q)}=\arg \min \left\|\alpha^{(q)}\right\|_{0} \text { s.t. } \mathbf{F}_{y m \_s u b}(q)=\Psi_{\tau_{-} \text {sub }} \alpha^{(q)}
$$

To effectively solve the problem, we utilize a sparse matrix recovery algorithm. Its pseudocode is described in Algorithm 2, which can be seen as another way of using EOMP. Similarly, the matrix $\boldsymbol{\alpha}=\left[\boldsymbol{\alpha} \wedge^{(1)}, \cdots, \boldsymbol{\alpha} \wedge \wedge^{(q)}, \cdots\right.$, $\left.\boldsymbol{\alpha} \wedge{ }^{\left(M_{\text {grid }}\right)}\right]^{T}$ is rewritten as $\mathbf{I}_{y m}\left(q, n^{\prime}\right)$.

The signal processed by the reconstruction algorithm can be expressed as

$$
\mathbf{I}_{y m}\left(q, n^{\prime}\right)= \begin{cases}A_{k}, & q=q_{k}, n^{\prime}=n_{k}^{\prime}, \\ 0, & \text { others. }\end{cases}
$$

Obviously, (25) is the distribution of the scattering coefficient in the time-delay Doppler grid. Therefore, based on the above analysis, we can see that the range-Doppler map for passive radar can be obtained by using the proposed CS-based processing method.

3.4. Summary of Method Flow. In order to intuitively show the processing technique, the flowchart of the proposed method is shown in Figure 4. The steps are briefly summarized as follows.

Step 1. After segmentation of reference signal and surveillance signal, perform FFT on $x(m, n)$ and $y(m, n)$ in the fast time $n$ direction, respectively.

Step 2. According to $\Phi_{m}$, construct Doppler dictionary $\Psi_{f \_s u b}$ by using the reference signal of the range-frequency domain and observation vector $\mathbf{F}_{y n_{\text {_sub }}}$ by using the surveillance signal of the range-frequency domain, and then perform
TABLE 5: Run times for different CS-PR methods.

\begin{tabular}{lcc}
\hline Methods & Full samples & Partial samples \\
\hline Original method & $3.365 \mathrm{~s}$ & $0.615 \mathrm{~s}$ \\
Feng et al.'s method & $0.069 \mathrm{~s}$ & $0.054 \mathrm{~s}$ \\
Proposed method & $0.161 \mathrm{~s}$ & $0.123 \mathrm{~s}$ \\
\hline
\end{tabular}

Algorithm 1 (MMV-OMP) to reconstruct Doppler frequency distribution.

Step 3. According to $\boldsymbol{\Phi}_{n}$, construct time-delay dictionary $\Psi_{\tau_{-} \text {sub }}$ and observation matrix $\mathbf{F}_{y m_{-} \text {sub }}$, and then perform Algorithm 2 (EOMP) to reconstruct range-Doppler map.

There are two points which remain to be explained. In Step 2, we actually only need to process $N_{\text {sub }}$ rangefrequency bins related to $\Phi_{n}$. In addition, the grid sizes $\left(\Delta f_{q}, \Delta_{\tau}{ }^{\prime}\right)$ and grid numbers $\left(M_{\text {grid }}, N_{\text {grid }}\right)$ of dictionaries $\left(\Psi_{f_{-} \text {sub }}, \Psi_{\tau_{-} \text {sub }}\right)$ can be set in accordance with the actual condition.

3.5. Comparative Analysis. We compare the proposed method with the time-delay/Doppler combination dictionary-based CS-PR method (named as original method here) presented in $[17,18]$ and Feng et al.'s method presented in [19]. Table 1 demonstrates the signal model, dictionary size of the original method, Feng et al.'s method, and the proposed method, respectively.

The major difference among these three methods lies in the signal model and method implementation. In the original method, the reflectivity map matrix is reconstructed by a cascade of 1-D CS reconstruction. All measurements are stacked into a single observation vector $\mathbf{y}_{\mathrm{vec}}$, the state of each timedelay/Doppler combination is stacked into the state vector $\boldsymbol{\alpha}_{\text {vec }}$, and the dictionary $\boldsymbol{\Psi}_{r D}$ by discretizing the delay Doppler plane on a grid takes up the most memory. The size is $M_{\text {sub }}$ $N_{\text {sub }} \times M_{\text {grid }} N_{\text {grid }}$, which leads to the memory occupation being too large and the computational burden being huge.

In both Feng et al.'s method and the proposed algorithm, the range reconstruction and the Doppler reconstruction are separately completed, which means that the dictionaries $\Psi_{D}$, $\Psi_{f \_ \text {sub }}^{(l)}, \Psi_{\tau_{-} \text {sub }}$ have much smaller size. The difference between the two methods is that the former only uses CS to estimate Doppler frequency while the latter uses CS in both directions, even though the use of EOMP is sameness. We can obtain high-resolution capability in the range coordinate. This is just what Feng et al.'s method does not have.

In addition, we can consider the fact that the input of EOMP is the output of MMV-OMP, which records the support set information. Therefore, the input data size of EOMP can be adjusted according to the size of the support set. As shown in Figure 5, there are two execution modes to obtain the range-Doppler map. The second execution mode can further reduce the computational burden due to the small amount of data. 

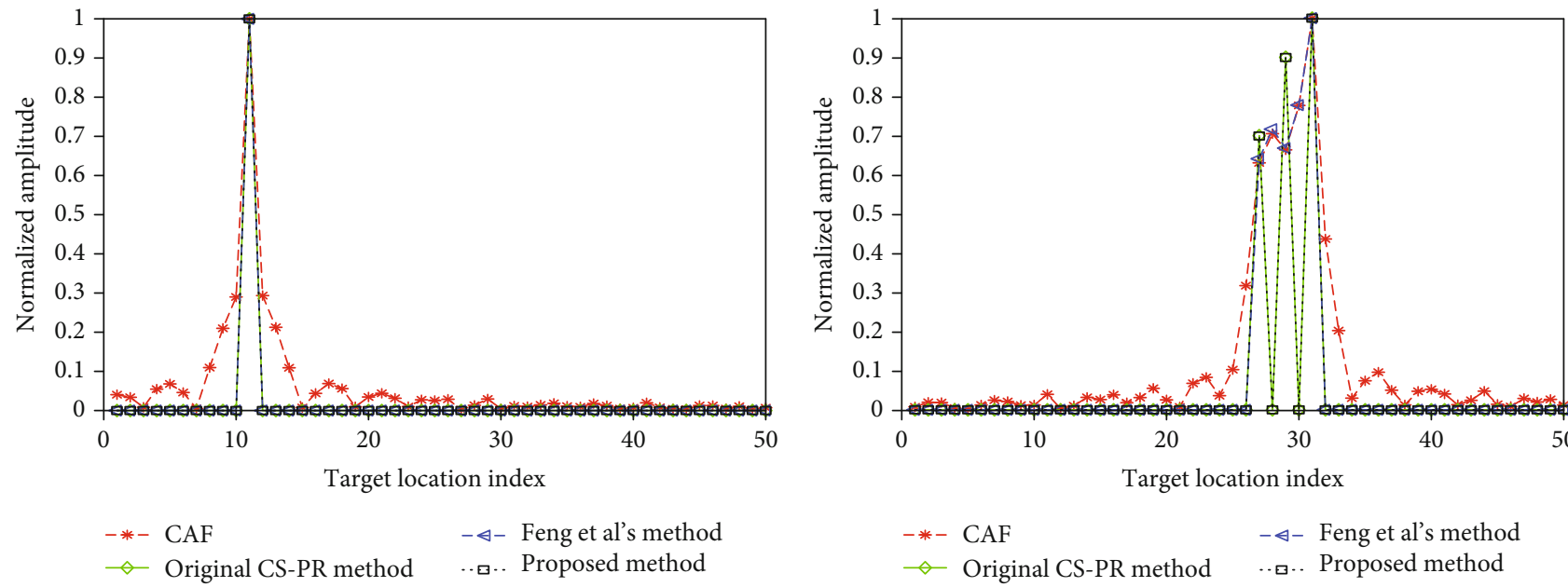

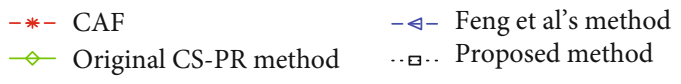

(a) (b)

FIGURE 8: Range profiles obtained by different methods: (a) target 1; (b) target 2.

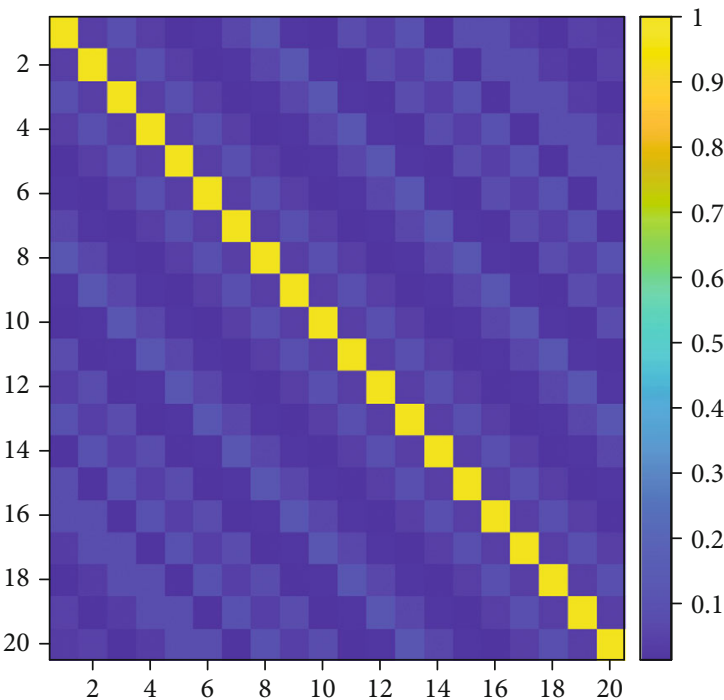

(a)

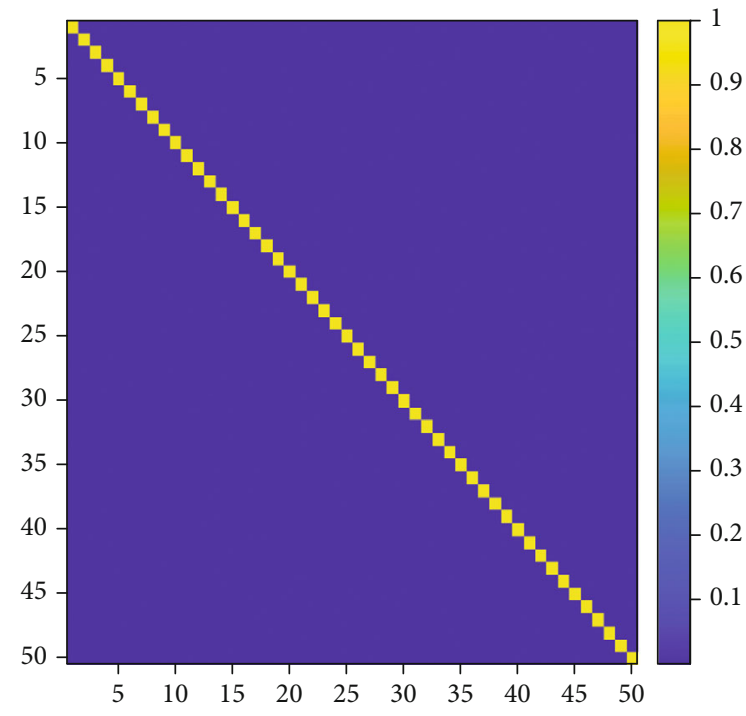

(b)

FIgURE 9: Gram matrices of original dictionaries $\left(\Delta f_{q}=\Delta f, \Delta_{\tau}{ }^{\prime}=\Delta_{\tau}\right)$ : (a) $\mathbf{G}_{\mathbf{f}}$; (b) $\mathbf{G}_{\tau}$.

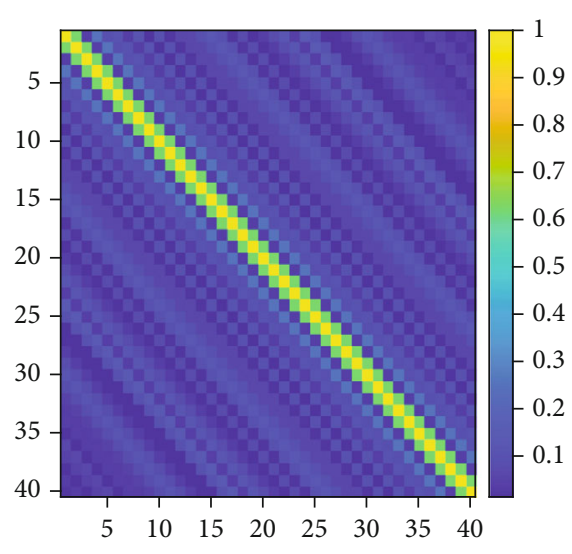

(a)

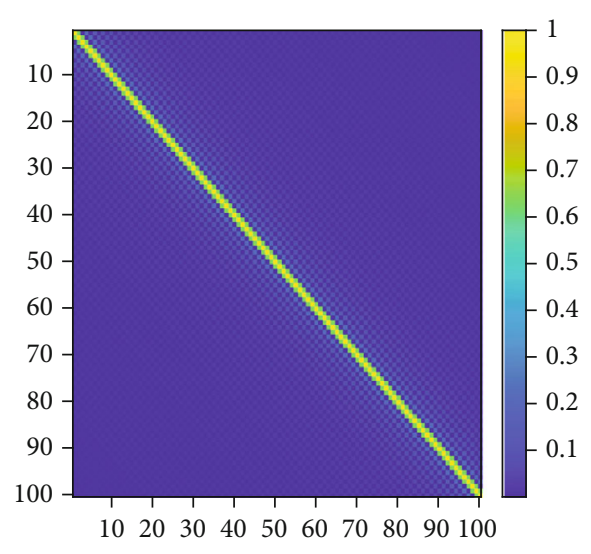

(b)

Figure 10: Gram matrices of refined dictionaries $\left(\Delta f_{q}=\Delta f / 2, \Delta_{\tau}{ }^{\prime}=\Delta_{\tau} / 2\right)$ : (a) $\mathbf{G}_{\mathbf{f}} ;$ (b) $\mathbf{G}_{\tau}$. 


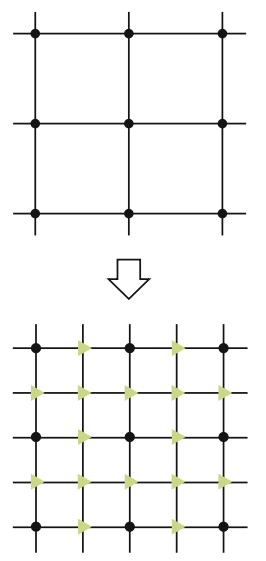

(a)

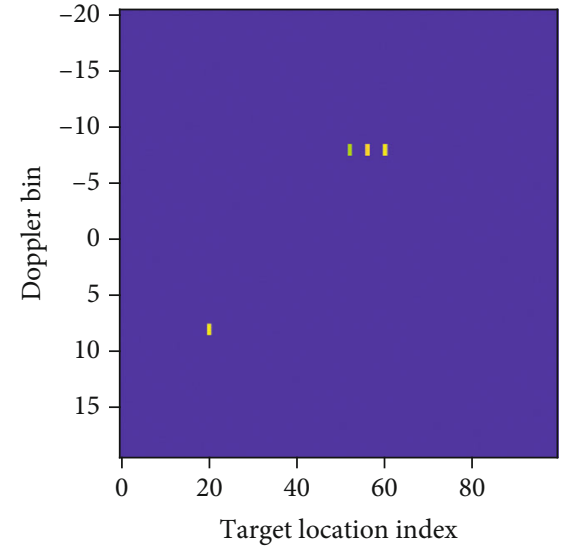

(b)

FIGURE 11: Result obtained by the proposed method with fine grid: (a) refining grid with $2 \times 2$; (b) RD map.

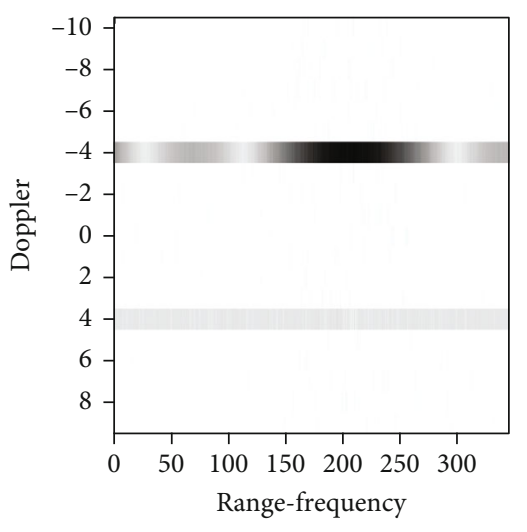

(a)

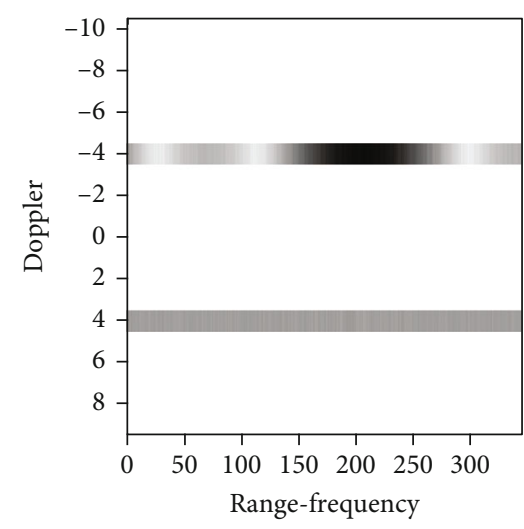

(b)

FIgURE 12: Results of sparse reconstruction of Doppler domain (SNR = $10 \mathrm{~dB}$ ) by (a) SMV-OMP and (b) MMV-OMP.

\section{Experimental Results}

In this section, we present experimental results with simulated data and real data. The effectiveness of the proposed processing method is demonstrated.

4.1. Simulation Data. We have conducted numerical experiments to investigate the performance of the proposed processing method. A digital television terrestrial broadcasting (DTTB) signal is simulated. The frame structure of signal is shown in Table 2, which includes frame header (945 symbols) and frame body (3780 symbols).

The parameters used in the simulation experiment are shown in Tables 3 and 4 . In the observation scene, the reflection mechanisms are assumed to be a point-like target (target 1) and a line-like target (target 2). Target 2 consists of three scatterer points, which are located in the same Doppler bin and become neighbors in the range direction.

At the beginning, the result obtained by the classical CAF is shown in Figure 6. As can be seen from Figures 6(a) and 6(b), the CAF can achieve the target scene recovery, but the reconstructed map is out of clarity due to the large sidelobes.
On the contrary, the CS-PR methods can be used to remove the sidelobes. The processing results by using the CS-PR methods listed in Table 1 are shown in Figure 7, which are the RD maps obtained by using full samples and partial samples from the same scenario. For the partial sample case, in order to ensure the same amount of data, the original method and the proposed method perform one-half data undersampling processing in the range direction and Doppler direction, respectively, while Feng et al.'s method only performs one-quarter data undersampling processing in the Doppler direction.

Figure 7 demonstrates that these processing methods based on CS can effectively suppress the sidelobe. The run times of different methods are summarized in Table 5. Apparently, the original CS-PR method uses much longer running time than the other two methods, due to the usage of the dictionary by the discretizing $\mathrm{RD}$ plane. In order to more intuitively observe the effect of different methods on the sidelobe suppression, the range profiles obtained by different methods are shown in Figure 8. From the figures, it shows that Feng et al.'s method cannot clearly identify the scatterer points in the range direction. 


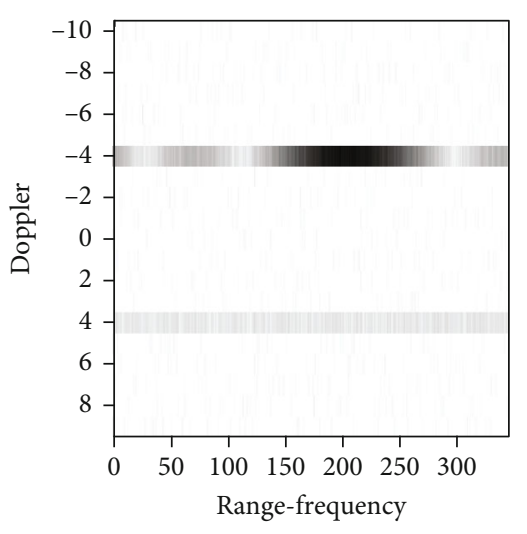

(a)

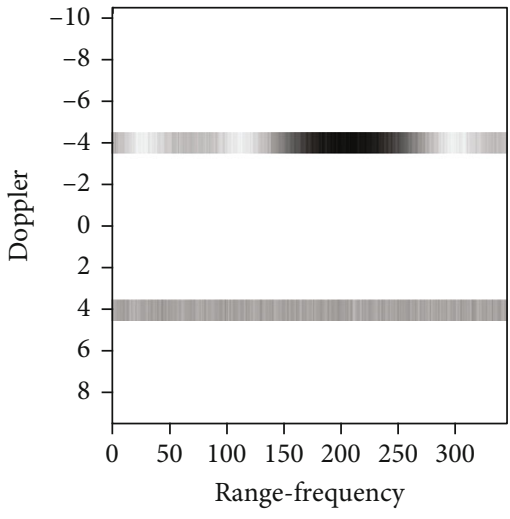

(b)

FIGURE 13: Results of sparse reconstruction of Doppler domain (SNR = $0 \mathrm{~dB}$ ) by (a) SMV-OMP and (b) MMV-OMP.

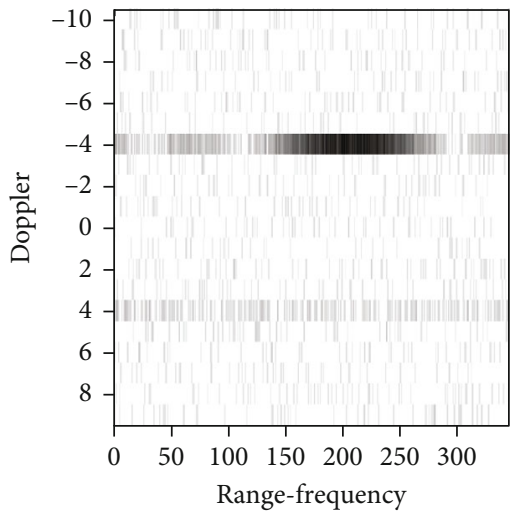

(a)

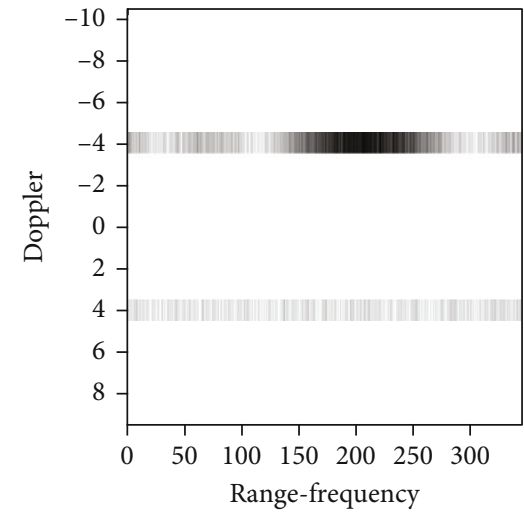

(b)

Figure 14: Results of sparse reconstruction of Doppler domain (SNR $=-10 \mathrm{~dB}$ ) by (a) SMV-OMP and (b) MMV-OMP.

It indicates that the range resolution of the method is limited, even though its run time is the least. Fortunately, the proposed processing method can reconstruct the target scene with a good performance both in accuracy and in computational efficiency.

It is known that the CS approach offers great potential for better resolution by using a finer dictionary. The grid sizes are set to be the same as CAF in previous experiments $\left(\Delta f_{q}=\Delta f, \Delta_{\tau}{ }^{\prime}=\Delta_{\tau}\right)$. Now, the Doppler grid and time-delay grid are only half the original size $\left(\Delta f_{q}=\Delta f / 2, \Delta_{\tau}{ }^{\prime}=\Delta_{\tau} / 2\right)$. As the grid is refined, the challenge is that the dictionary correlation will increase, which may lead to the performance degradation for CS. The Gram matrix is used to verify the dictionary coherence, and results are depicted in Figures 9 and 10. $\mathbf{G}_{\mathbf{f}}=\Psi_{f_{-} \text {sub }}^{H} \boldsymbol{\Psi}_{f_{\text {s sub }}}$ and $\mathbf{G}_{\tau}=\boldsymbol{\Psi}_{\tau_{-} \text {sub }}^{H} \boldsymbol{\Psi}_{\tau_{-} \text {sub }}$ represent Gram matrices in Doppler direction and range direction, respectively. As can be seen from Figures 9 and 10, each Gram matrix is close to the unit matrix. Due to this coherence characteristic, CS is able to produce superresolution radar images. Figure 11 shows the RD map obtained by the proposed method when the grid is refined. It is observed that the resolution improvement is achieved by utilizing a fine grid.
In order to demonstrate the advantages of OMP under the MMV model, we compare the Doppler reconstructions by SMV-OMP and MMV-OMP at different signal-to-noise ratios (SNR). The values of SNR are $10 \mathrm{~dB}, 0 \mathrm{~dB}$, and -10 $\mathrm{dB}$. The parameters of target 1 use the following settings. The location index is 10, the Doppler bin is 4 , and the amplitude is 0.3 . Other parameters remain unchanged. The results are shown in Figures 12-14. For SMV-OMP, there are a number of Doppler reconstruction errors, which will lead to insufficient energy accumulation in the range direction. Figure 15 shows the final $\mathrm{RD}$ maps when $\mathrm{SNR}$ is $-10 \mathrm{~dB}$. It can be found that target 1 is not visible in the RD map obtained by SMV-OMP. On the contrary, MMV-OMP has robust performance because it considers the information of multiple observations.

4.2. Real Data. We use real data from an DTV-based PR system to further verify the proposed method. The parameters used are shown in Table 6. We obtain results for CAF and the proposed method. The RD map obtained by the CAF approach is shown in Figure 16; Figures 17 and 18 show the results of CS reconstruction if $25 \%$ of the full data is used. In comparison to the CAF, the proposed method shows good performance owing to CS. 


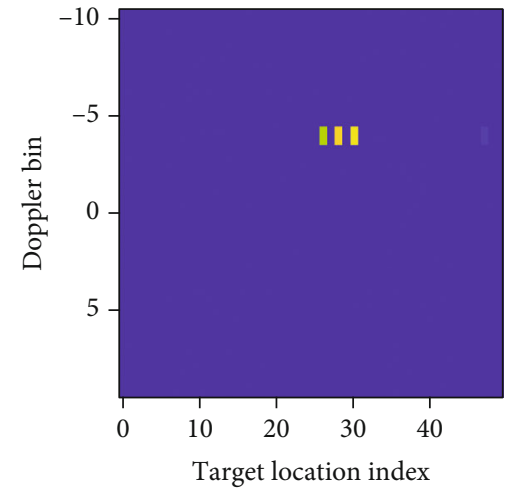

(a)

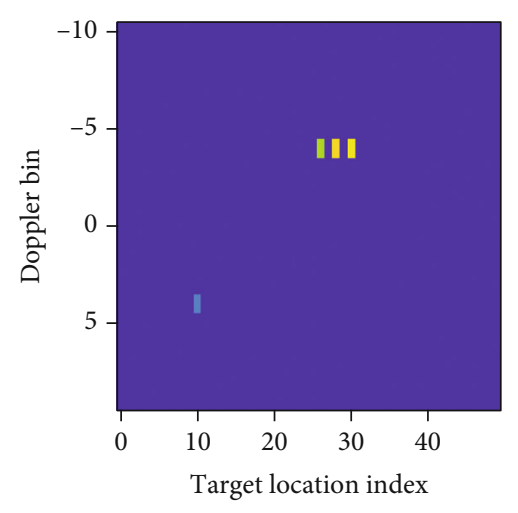

(b)

FIGURE 15: RD maps (SNR $=-10 \mathrm{~dB}$ ) obtained by (a) SMV-OMP and (b) MMV-OMP.

TABLE 6: System parameters.

\begin{tabular}{lcc}
\hline Parameters & Symbol & Value \\
\hline Carrier frequency & $f_{c}$ & $674 \mathrm{MHz}$ \\
Bandwidth & $B$ & $7.56 \mathrm{MHz}$ \\
Sample frequency & $f_{s}$ & $10 \mathrm{MHz}$ \\
Number of segments & $M$ & 2048 \\
Sample number & $N$ & 2048 \\
Number of range grid & $M_{\text {grid }}$ & 50 \\
Number of Doppler grid & $N_{\text {grid }}$ & 200 \\
\hline
\end{tabular}

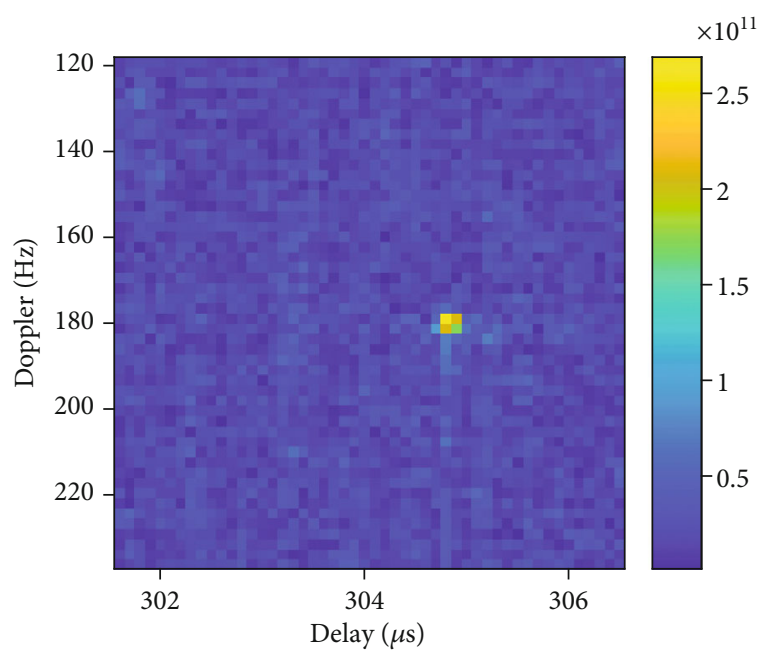

Figure 16: RD map obtained by CAF.

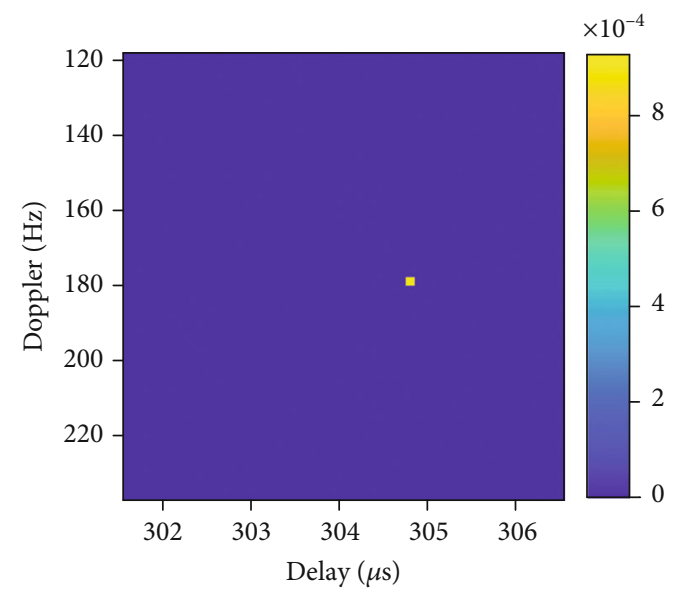

FIGURE 17: RD map obtained by the proposed method.

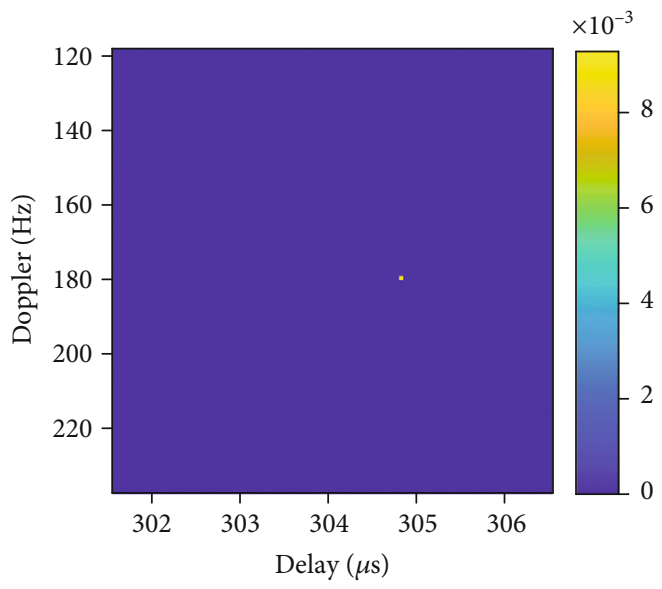

FIGURE 18: RD map obtained by the proposed method with fine grid. 


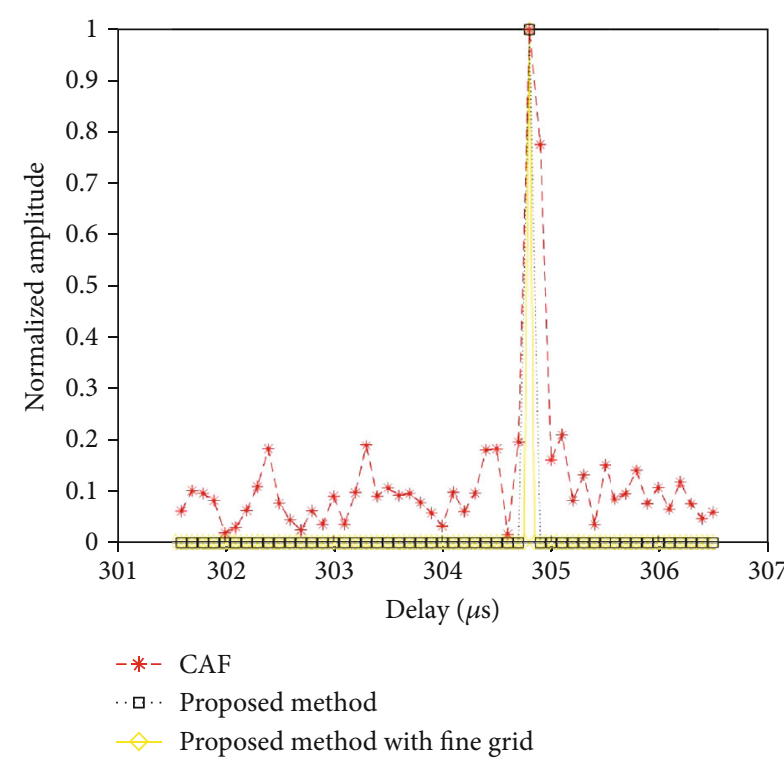

(a)

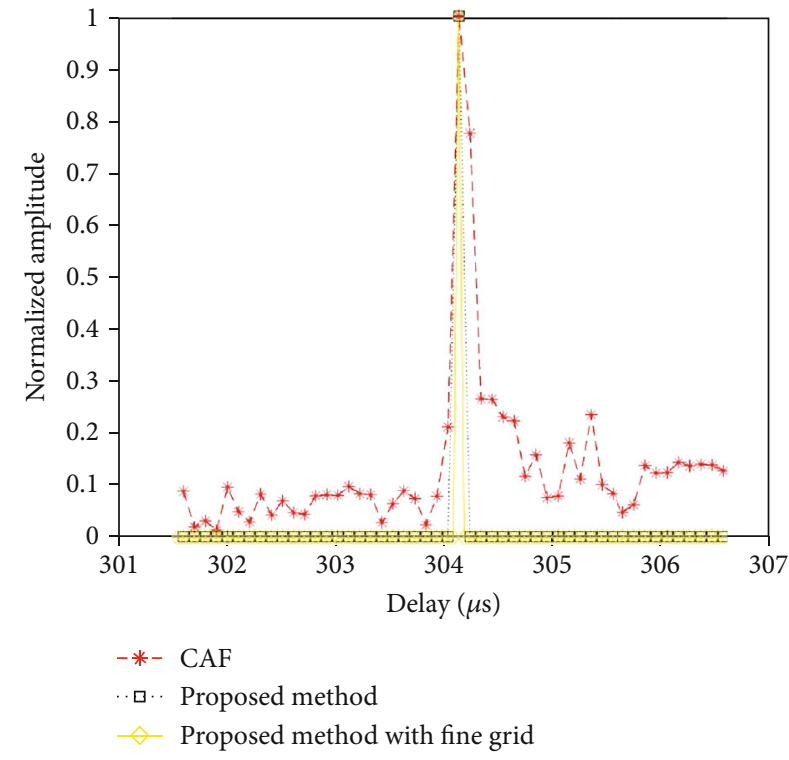

(b)

Figure 19: Profiles of target: (a) in range direction; (b) in Doppler direction.

For a more illustrative comparison, two cuts are made along the location of the target in the range and Doppler directions shown in Figures 19(a) and 19(b). The proposed method has a considerably lower sidelobe level than the CAF and indeed allows for improved range and Doppler resolutions by the fine gridding.

\section{Conclusion}

In this paper, the problem of compressed sensing-based range-Doppler processing for passive radar is investigated. In order to reduce the sidelobes and improve the resolution, we have proposed a novel CS-PR method, in which the modified MMV-OMP algorithm is used to perform sparse reconstruction of Doppler dimension, and then, the EOMP algorithm is used to perform sparse reconstruction of range dimension. Compared to previous CS-PR methods, we can achieve a high-quality reconstruction of the range-Doppler map of target scenario and do not suffer from the heavy computational burden. The effectiveness of the proposed method is verified by experiments with simulated data and real data. The improved resolution capability will be helpful to widen the extent of application.

\section{Data Availability}

The data that support the findings of this study are available from the corresponding author upon reasonable request.

\section{Conflicts of Interest}

The authors declare that they have no competing interests.

\section{Acknowledgments}

The authors are grateful to the National Science Foundation of China (Grant 61771046, 61931015, and 61731023) and the Beijing Natural Science Foundation (L191004) for their support of this research.

\section{References}

[1] P. Howland, "Passive radar systems," IEE Proceedings-Radar, Sonar and Navigation, vol. 152, no. 3, pp. 105-106, 2005.

[2] A. Lauri, F. Colone, R. Cardinali, C. Bongioanni, and P. Lombardo, "Analysis and emulation of FM radio signals for passive radar," in 2007 IEEE Aerospace Conference, pp. 110, Big Sky, MT, 2007.

[3] L. Wan, X. Kong, and F. Xia, "Joint range-Doppler-angle estimation for intelligent tracking of moving aerial targets," IEEE Internet of Things Journal, vol. 5, no. 3, pp. 1625-1636, 2018.

[4] D. K. P. Tan, H. Sun, Y. Lu, M. Lesturgie, and H. L. Chan, "Passive radar using global system for mobile communication signal: theory, implementation and measurements," IEE Proceedings. Part F, Radar, Sonar and Navigation, vol. 152, no. 3, pp. 116-123, 2005.

[5] J. E. Palmer, H. A. Harms, S. J. Searle, and L. Davis, "DVB-T passive radar signal processing," IEEE Transactions on Signal Processing, vol. 61, no. 8, pp. 2116-2126, 2013.

[6] Z. Gao, R. Tao, Y. Ma, and T. Shao, "DVB-T signal crossambiguity functions improvement for passive radar," in 2006 CIE International Conference on Radar, pp. 1-4, Shanghai, 2006.

[7] Y. D. Zhang, M. G. Amin, and B. Himed, "Structure-aware sparse reconstruction and applications to passive multi-static radar," IEEE Aerospace and Electronic Systems Magazine, vol. 32, no. 2, pp. 68-78, 2017.

[8] A. S. Tasdelen and H. Koymen, "Range resolution improvement in passive coherent location radar systems using multiple 
FM radio channels," in 2006 IET Seminar on Waveform Diversity and Design in Communications, Radar and Sonar, pp. 2331, London, 2006.

[9] K. E. Olsen and K. Woodbridge, "Analysis of the performance of a multiband passive bistatic radar processing scheme," in 2010 International Waveform Diversity and Design Conference, pp. 000142-000149, Niagara Falls, ON, 2010.

[10] K. E. Olsen and K. Woodbridge, "Multiband passive bistatic DVB-T radar range resolution improvements and implications," in 2012 13th International Radar Symposium, pp. 2831, Warsaw, 2012.

[11] Y. Feng, T. Shan, Z. Zhuo, and R. Tao, “The migration compensation methods for DTV based passive radar," in 2013 IEEE Radar Conference (RadarCon13), pp. 1-4, Ottawa, ON, 2013.

[12] K. E. Olsen and J. M. Christiansen, "Multiband passive bistatic radar coherent range and Doppler-walk compensation," in 2015 IEEE Radar Conference (RadarCon), pp. 0123-0126, Arlington, VA, 2015.

[13] D. L. Donoho, "Compressed sensing," IEEE Transactions on Information Theory, vol. 52, no. 4, pp. 1289-1306, 2006.

[14] E. J. Candes and M. B. Wakin, "An introduction to compressive sampling," IEEE Signal Processing Magazine, vol. 25, no. 2, pp. 21-30, 2008.

[15] M. A. Herman and T. Strohmer, "High-resolution radar via compressed sensing," IEEE Transactions on Signal Processing, vol. 57, no. 6, pp. 2275-2284, 2009.

[16] J. Ender, "A brief review of compressive sensing applied to radar," in 2013 14th International Radar Symposium (IRS), pp. 3-16, Dresden, 2013.

[17] M. Wei, "Compressive sensing for passive surveillance radar using DAB signals," in 2014 International Radar Conference, pp. 1-6, Lille, 2014.

[18] M. N. Tabassum, M. A. Hadi, and S. Alshebeili, "Compressive sensing based passive bistatic radar processing using timedomain complex data," in IEEE International Symposium on Signal Processing and Information Technology (ISSPIT), pp. 63-68, Abu Dhabi, United Arab, 2015.

[19] W. Feng, J. Friedt, G. Cherniak, and M. Sato, "Batch compressive sensing for passive radar range-Doppler map generation," IEEE Transactions on Aerospace and Electronic Systems, vol. 55, no. 6, pp. 3090-3102, 2019.

[20] S. F. Cotter, B. D. Rao, Kjersti Engan, and K. Kreutz-Delgado, "Sparse solutions to linear inverse problems with multiple measurement vectors," IEEE Transactions on Signal Processing, vol. 53, no. 7, pp. 2477-2488, 2005.

[21] R. Cardinali, F. Colone, C. Ferretti, and P. Lombardo, "Comparison of clutter and multipath cancellation techniques for passive radar," in 2007 IEEE Radar Conference, pp. 469-474, Boston, MA, 2007.

[22] J. E. Palmer and S. J. Searle, "Evaluation of adaptive filter algorithms for clutter cancellation in passive bistatic radar," in 2012 IEEE Radar Conference, pp. 0493-0498, Atlanta, GA, 2012.

[23] J. L. Garry, G. E. Smith, and C. J. Baker, "Direct signal suppression schemes for passive radar," in 2015 Signal Processing Symposium (SPSympo), pp. 1-5, Debe, 2015.

[24] T. Peto and R. Seller, "Time domain filter comparison in passive radar systems," in 2017 18th International Radar Symposium (IRS), pp. 1-10, Prague, 2017.
[25] A. Filip-Dhaubhadel and D. Shutin, "Long coherent integration in passive radar systems using super-resolution sparse Bayesian learning," IEEE Transactions on Aerospace and Electronic Systems, vol. 57, no. 1, pp. 554-572, 2020.

[26] C. Moscardini, D. Petri, A. Capria, M. Conti, M. Martorella, and F. Berizzi, "Batches algorithm for passive radar: a theoretical analysis," IEEE Transactions on Aerospace and Electronic Systems, vol. 51, no. 2, pp. 1475-1487, 2015. 